Luisa Maria Nieto Ramirez ${ }^{1 \uparrow}$, Beatriz E. Ferro ${ }^{2 *}$, Gustavo Diaz ${ }^{3,4}$, Richard M. Anthony ${ }^{5}$,

1. Universidad Santiago de Cali, Cali, Colombia.

2. Departamento de Salud Pública y Medicina Comunitaria, Universidad Icesi, Cali, Colombia.

4. Universidad Icesi, Cali, Colombia.

5. Mycobacteria Diagnostic Laboratory for Bacteriology and Parasitology (BPD)

3. Centro Internacional de Entrenamiento e Investigaciones Médicas (CIDEIM), Center for Infectious Disease Research, Diagnostics and Perinatal Screening (IDS) National Institute for Public Health and the Environment (RIVM), Bilthoven, The Netherlands.

6. University of Groningen, University Medical Center Groningen, Department of Medical Microbiology, Groningen, The Netherlands.

7. Department of Medical Microbiology, Radboud University Medical Center,

\title{
$21{ }^{*}$ Corresponding author
}

22 E-mail: beferro@gmail.com (BEF)

23 TThese authors contributed equally to this work. 


\section{Abstract}

Beijing strains of Mycobacterium tuberculosis (lineage2) have been associated with drug-resistance and transmission of tuberculosis worldwide. Most of the Beijing strains identified in the Colombian pacific coast exhibited a multidrug resistant (MDR) phenotype, in contrast with the phenotype observed in Beijing isolates from other SouthAmerican countries. We wanted to evaluate the clonality and genetic background of the Beijing strains isolated in Colombia that belong mostly to the spoligo-international type (SIT) 190. Out of 37 Beijing stains characterized in an 8-years period, we identified five Beijing clones; 36 that belong to the SIT190 type and only one to SIT1. Two loci in VNTR typing: MIRU 39 and QUB11b exhibited the highest level of variation among these strains. Out of the 37 Beijing strains, only one was drug susceptible, 28 represented MDR-TB, four extensively drug resistant TB (XDR-TB) and four pre-XDRTB. The mutations rpoB S531L and katG S315T1 were the most common among the MDR strains as reported elsewhere. Whole genome sequencing analysis allowed us to classify them as modern lineage Beijing strains, sharing up to 76 out of the 275 SNPs described in Beijing strains, as identified worldwide by Schürch et al; including 54 nonsynonymous SNPs and 23 silent mutations. We were also able to confirm the presence of 8 specific SNPs that were so far only found in the Beijing strains from Colombia. The presence of modern Beijing strains, most of them representing MDR-TB, suggests a different origin of this $M$. tuberculosis lineage compared to other Beijing strains found in neighboring countries, such as Peru. The specific 8-SNP signature confirmed the identity of these Colombian Beijing MDR strains. This work may serve as a genetic 
47 baseline to study the evolution and spread of $M$. tuberculosis Beijing strains in

48 Colombia, which play an important role in the control of MDR-TB.

\section{Introduction}

The Beijing genotype is considered one of the most successful and virulent lineages of Mycobacterium tuberculosis (Mtb) (1-4). Tuberculosis (TB) is still the leading cause of death due to a single infectious agent worldwide, largely because of the contribution of drug resistant Mtb strains (5). In many settings, Beijing strains have not only been found to be significantly correlated with drug resistance, but also with active transmission of multidrug (MDR) and extensively drug resistant (XDR) TB $(1,2,6)$. Beijing is the major representative strain of lineage 2, also known as East-Asian lineage, which is defined by the deletion of region of difference (RD) RD105 (7). Two different sublineages of Beijing strains, the ancient and modern strains, have been described (also known as 'atypical' and 'typical' Beijing variants, respectively). The two variants show differences in distribution, drug resistance and virulence patterns. The ancient sub-lineage strains have been found predominantly in Russia, Korea and Japan (8-10), while modern sub-lineages are distributed worldwide, and have been largely associated with drug resistance and hypervirulence (11-13).

Although the prevalence of Beijing strains was not high in South America (14), several countries have now reported the presence of this genotype among clinical isolates $(9,15-18)$. The proportion of Beijing strains in Peru (close to $9 \%$ ) has increased in the last decade, with predominance of the modern sub-lineage (9); at the moment 
without association with drug resistance. Similarly, countries such as Paraguay and

70 Ecuador have reported Beijing strains only among pan-drug-susceptible cases.

We identified a cluster of 24 Beijing strains among MDR and XDR Mtb isolates

72 from Southwestern Colombia $(19,20)$. In a study conducted in Buenaventura- a port city

73 that is considered a MDR-TB hot-spot in Colombia- we found a high proportion of

74 Beijing strains (30\%) among MDR-TB isolates, we also found that $25 \%(18 / 73)$ of the of MDR-Mtb strains of the Beijing genotype (21) in this region. According to the SpolDB4 database, the Beijing strains observed in the Colombian pacific coast (that belong to the Spoligo-International-Type SIT 190), have been observed previously in

79 the United States, Japan, Cuba, among other countries (22). However, more discriminative molecular analysis is needed to better characterize the cluster detected in

81 Colombia to decipher possible epidemiological links between these cases and the worldwide spread of Beijing strains. Therefore, this study aimed to establish a comprehensive genetic profile of Beijing strains isolated in Colombia and to provide additional insights into its biology in comparison to Beijing strains from other geographical areas.

\section{Materials and Methods}

\section{Ethics Statement}

This study was approved by the Institutional Review Board of CIDEIM, which

91 authorized a waiver of informed consent from the human research subjects who 
92 provided the samples. Additionally, data was anonymously analyzed and confidentiality

93 preserved using codes instead of identifiable variables.

94

95

96

97

98

99

100

101

102

103

104

105

106

107

108

109

110

111

112

113

114

115

116

117

\section{Study Samples}

During the period 2002 to 2010, 651 Mtb isolates from Valle del Cauca, Colombia were sent to CIDEIM from public and private health institutions to perform routine drug susceptibility testing (DST) on basis of the proportion Middlebrook 7H10 agar method for both first (isoniazid, rifampicin, ethambutol) and second line antibiotics (amikacin, ciprofloxacin or moxifloxacin) (23). The strains were stored at $-80^{\circ} \mathrm{C}$. For this study 311 were thawed to perform genetic typing and complementary genetic analyses. In total, 37 Beijing strains were detected and used for further analysis, only one isolate per patient was included.

\section{Genetic typing}

We performed a molecular characterization of the 37 Mtb Beijing isolates using two Polymerase Chain Reaction (PCR) based methodologies: spoligotyping (24) and MIRU-VNTR (Mycobacteria Interspersed Repetitive Unit - Variable Number Tandem Repeat) 24 loci typing (25). DNA was extracted using the CTAB method (26). Agarose gel electrophoresis was performed to determine the number of repeats for each locus.

Finally, allelic assignation was done using Quantity one software (Biorad®) to determine the length of the PCR products for each of the 24 loci analyzed. External quality control was assessed through the participation in the second worldwide proficiency study of MIRU- VNTR (27).

\section{Detection of mutations associated with drug resistance}


DNA was extracted according to the manufacturer's instructions (HainLifescience

$119 \mathrm{GmbH}$, Nehren, Germany). Mutations associated with resistance to first- and second-

120 line anti-tuberculosis drugs were detected using GenoType ${ }^{2}$ MTBDRplus and s/ assays

121 (HainLifescience, Nehren, GmbH, Germany) respectively.

122

123

124

125

126

127

128

130

131

132

133

134

135

\section{Beijing strains classification}

In order to confirm that the strains belonged to the Beijing genotype family, further analyses were performed using 10 representative strains of each branch of the dendrogram based on the spoligotyping and MIRU-VNTR 24 loci typing analysis. These analyses included the evaluation of the genomic region of difference 105 (RD 105), which presence is a genetic marker for the Beijing genotype. Additionally, we performed a SNP analysis of the $f b p B-238$ region, to classify the selected Beijing strains into the Vietnam (V) "typical" (+) or atypical (-) sub-lineage, according to the scheme of Schürch et al (28), using a Multiplex Ligation-dependent Probe Amplification (MLPA) assay with readout facilitated by the Luminex bead technology, as described previously (29). The MLPA analysis was conducted by the Royal Tropical Institute (KIT) in The Netherlands and was also used to confirm the MIRU and the mutation analysis in the katG, inhA, rpoB, gyrA, rrs and embB resistance genes (30).

Two out of 37 Beijing strains were selected to be whole genome sequenced using Illumina sequence technology at The Broad Institute of MIT \& Harvard. Analysis of SRA files was done using CLC genomics workbench 12 software, having H37Rv as the reference sequence.

\section{Data Analysis}


The Spoligo-International-Type (SIT) number and the multiple locus VNTR

analysis (MLVA) using 15 of the most discriminatory loci (MLVA MtbC15-9) were

144 determined using the http://www.miru-vntrplus.org web page and SITVIT2 (database of

145 the Pasteur Institute of Guadeloupe). The information obtained was used to build the

146 phylogenetic analysis based on MIRU-VNTR patterns. VNTR typing results were

147 recorded as character data type and compared to database at the National Institute for

148 Public Health and the Environment (RIVM) in the Netherlands.

\section{Results}

The identity of all 37 Beijing isolates was confirmed using spoligotyping.

According to the DST, 30 of these strains were MDR-TB, 6 XDR-TB, and only one was found drug-susceptible. Patient's median age was 29 years (IQR: 24-40 years). Most strains were isolated from female $(23 / 37,62 \%)$ and pulmonary TB patients $(34 / 35$, $97.1 \%)$

All except one strain were typed as Spoligo-International number-SIT 190 (000000000003731). The remaining, susceptible strain, was typed as SIT1 (000000000003771). MIRU-VNTR analysis using 24 loci was used to explore further differences among the highly homogeneous SIT 190 cluster. However, only two loci (QUB11b and MIRU 39) were able to discriminate isolates in the SIT 190 cluster. Among those two loci, MIRU 39 revealed the highest differences, with 2, 3 and 4 repeats among the Beijing SIT190 strains. In addition, five more loci (Mtub39, Mtub04, MIRU 31, 27, and 40) showed different number of repeats between SIT 190 and SIT 1 strains (Fig 1). A phylogenetic analysis using both the spoligotyping and MIRU-VNTR 
24 loci analyses confirmed the classification of SIT190 and SIT1 as different clades and showed that among the SIT190 clade there were four different clones (Fig 1).

Fig 1. Dendrogram of the Beijing strains discriminated by MIRU VNTR 24 loci and Spoligotyping patterns. MLVA MtbC15 type for the 15 most discriminatory loci a MLVA MtbC 9 type for the 9 auxiliary loci defined by Supply et al, 2006 (25). ND: Information not available. DSP: Drug susceptibility profile.

\section{Detection of mutations associated with drug resistance}

In total 33 (29 MDR and 4 XDR) out of the 37 Beijing strains were successfully evaluated for the presence of mutations associated with first- and second-line drug resistance. The mutations S531 $\mathrm{L}$ in $r p o B$ and S315T in katG, associated with resistance to rifampicin and isoniazid, respectively, were the most frequently found as expected in MDR cases (Table 1). All four XDR strains were confirmed using the genotype analysis that also allowed the detection of four additional pre-XDR strains (defined as MDR strain that is also resistant to either a fluoroquinolone or a second-line injectable) (31).

The ethambutol associated mutation M306V in the embB gene was also the most frequently encountered among the ethambutol resistant strains (Table 1). 
Table 1. Frequency of mutations associated with drug resistance in Beijing genotype family strains from Colombia.

Gene mutation probe (mutation)

$\%$ of strains (\# of mutated strains/\# of

resistant strains detected by

GenoTypeMTBDRplus and MTBDRsl)

\begin{tabular}{lc}
\hline rpoBMUT3 (S531L) & $97(32 / 33)$ \\
katGMUT1 (S315T1) & $100(33 / 33)$ \\
inhAMUT1 (C15T) & $3(1 / 33)$ \\
gyrAMUT3C (D94G) & $13(4 / 30)^{a}$ \\
rrsMUT1 (A1401G) & $29(8 / 28)$ \\
embBMUT1B (M306V) & $91(29 / 32)$ \\
\hline
\end{tabular}

a One strain had double mutations MUT3A and MUT3C.

192

193

194

195

196

197

198

199

200

201

202

203

204

205

206

\section{Beijing strains classification}

The East/Asian RD105 deletion was present in all the subset of 10 Beijing strains, confirming their identification as members of the Beijing lineage. Moreover, all the strains tested, including the SIT 1 and SIT 190, were further classified into the Beijing sublineage $\mathrm{V}+$.

\section{Whole genome sequencing analysis}

The whole genome sequences of two Beijing strains from this study, designated as 38088 (fully susceptible) and 38765 (MDR), are available at the National Center for Bioinformatic Information (NCBI) with the SRA numbers SRS565195 and SRS565201 respectively. Based on the 275 SNPs proposed by Schürch et al., (28), these two Beijing strains isolated in Southwestern Colombia exhibited features of a modern lineage, such as the mutation at the gene mutT2 Gly58Arg (transversion C/G at position 1286766), confirming our previous Beijing strain classification. Strain 38088 

revealed the presence of 8 additional SNPs among Beijing MDR strains (Table 3). Also,

21138088 and 38765 strains have an insertion of a $G$ at the position $1406760^{\wedge} 1406761$ in

212 the Rv1258c gene.

Table 2. Genes (location) with single nucleotide polymorphism shared between Colombian Beijing strains and other Beijing strains characterized worldwide.

\begin{tabular}{|c|c|}
\hline \multicolumn{2}{|c|}{ SNP Location } \\
\hline Non-synonymous $(n=54)$ & Silent $(n=23)$ \\
\hline $\begin{array}{l}\text { recF, fadD34, oxyS, ccsA, recD, echA4, } \\
\text { rpsL*, far, mutT2, narJ, fadD6, alkA, } \\
\text { uvrC (1594906), uvrC (1595342), ansA, } \\
\text { polA (1831220), polA (1831226), lysS, } \\
\text { moeX, secA2, glcB, katG**, rpmG, } \\
\text { pknJ, pepE, uspA, accD1, recX, fdhD, } \\
\text { ligB, nudC, maf, sseA, esxU, rmIC, otsA, } \\
\text { mce4C, fadD19, Itp3, nth, ponA2, dnaQ } \\
\text { (4156099), dnaQ (4156503), dnaQ } \\
\text { (4155748), recR, ligC, dppB, ponA2, } \\
\text { recR, cut5b, ligC, fadE36, embA, papA2, } \\
\text { glpQ1, mycP2, 4393590. }\end{array}$ & $\begin{array}{c}\text { acs, cobG, cobQ2, ctpG, } \\
\text { cysA3***, dnaZX, echA21, } \\
\text { embB, fadB4, fba, fusA2, } \\
\text { hemB, ligC, IprK, murC, ogt, } \\
\text { polA, pyrG, radA, recC, } \\
\text { sigM, tatD, tpiA. }\end{array}$ \\
\hline
\end{tabular}


Table 3. Comparison of the previously reported SNPs in Beijing-like strains from Colombia and the MDR Beijing strains of this study

\begin{tabular}{|c|c|c|c|c|c|c|}
\hline $\begin{array}{c}\text { Accession } \\
\text { number }\end{array}$ & $\begin{array}{l}\text { Gene } \\
\text { name }\end{array}$ & Position & $\begin{array}{c}\text { Reference } \\
\text { H37Rv }\end{array}$ & $\begin{array}{c}\text { Mutation } \\
\text { found in } \\
\text { Beijing strains } \\
\text { from } \\
\text { Colombia (32) }\end{array}$ & $\begin{array}{c}\text { Mutation found } \\
\text { in MDR Beijing } \\
\text { strain } 38765\end{array}$ & $\begin{array}{c}\text { Mutation } \\
\text { found in FS } \\
\text { Beijing strain } \\
38088\end{array}$ \\
\hline Rv0355c & PPE8 & 434226 & A & Deletion & Wild type & Wild type \\
\hline Rv0355c & PPE8 & 434227 & C & Wild type & Deletion & Wild type \\
\hline Rv0197 & Rv0197 & 233949 & C & $\mathrm{T}$ & $\mathrm{T}$ & Wild type \\
\hline Rv0753c & $m m s A$ & 845542 & G & A & A & Wild type \\
\hline Rv0988 & Rv0988 & 1106176 & $A$ & C & C & Wild type \\
\hline Rv1723 & $R v 1723$ & 1950068 & C & $\mathrm{T}$ & $\mathrm{T}$ & Wild type \\
\hline Rv2308 & $R v 2308$ & 2580877 & G & $\mathrm{T}$ & $\mathrm{T}$ & $\mathrm{T}$ \\
\hline Rv2940c & $R v 2940 c$ & 3279637 & G & $A$ & A & Wild type \\
\hline Rv3806c & ubiA & 4269304 & $A$ & C & C & Wild type \\
\hline Rv3862c & whiB6 & 4338365 & A & $\mathrm{G} / \mathrm{C}$ & G & Wild type \\
\hline
\end{tabular}

FS: fully susceptible

\section{Discussion}

In this study, we established the genetic profile of a cluster of 37 Beijing strains isolated in Colombia and compared it with Beijing strains circulating elsewhere in the world. Five interesting findings are reported: first, the conserved genetic make-up of Beijing strains isolated in Colombia confirmed our previous observation of the active and recent transmission of Beijing strains in Colombia, particularly in Buenaventura, where the main port on the Colombian pacific coast is located (19). Second, the large majority of the Beijing strains (36/37) contained mutations associated with MDR- or XDR-TB profiles, again confirming the association between Beijing and drug resistance 
233 in Colombia. Third, these Beijing strains formed a genetically highly conserved cluster,

234 where four branches were separated, mainly based on the discriminatory capacity of

235 24-loci MIRU-VNTR typing (particularly two loci: 39 and QUB11b). Fourth, there was

236 limited diversity in mutations associated with resistance in these Beijing strains, almost

237 all were MDR due to the presence of mutations most commonly described worldwide

238 (33-36). Finally, all the Beijing strains from this study belonged to the 'typical' or

239 'modern' sub-lineage, highly similar to the $\mathrm{V}+$ lineages earlier described by Schürch et al

240 (28), and commonly found in Vietnam. Our results support the "distribution" profile

241 previously determined for modern Beijing strains worldwide.

243 strains and the predominance of the modern sub-lineage in this country $(9,37)$. It has

244 been hypothesized that Beijing strains identified in Peru probably arrived from China,

245 when a significant Chinese migration took place, back in the 19th century (9). However,

246 the Beijing strains circulating in Peru are genotypically different compared to the ones

247 circulating in Colombia, indicating a different origin. The Beijing strains (mostly, SIT190)

248 isolated in Colombia have shown highly resistant phenotypes and this varies from the

249 Beijing strains isolated in Peru and other South American countries, where no

250 association with drug resistance has been established $(9,14)$. These findings support

251 the idea that different Beijing strains (or their ancestors) were introduced at different

252 occasions in Southern American countries, most likely reflecting the diversity in human

253 migration since ancient times (12). This is also in agreement with the findings of

254 Schürch et al on the introduction of multiple sources of spread of Beijing strains to

255 different geographical areas on many different occasions (28). However, it is important 
256 to highlight that the Colombian Beijing strains are genetically highly conserved and that

257 this may reflect recent introduction and spread.

Regarding the WGS analysis, there were additional SNPs that have been also

259 reported in other globally identified Beijing strains (28). For instance, both Beijing strains

260 (38088 and 38765) exhibited an insertion of a $G$ at the position $1406760^{\wedge} 1406761$ in

261 the Rv1258c gene, previously identified in modern Beijing strains from Guatemala (38).

262 Rv1258c encodes for an efflux pump that was previously proposed to be linked to

263 streptomycin resistance (39). SNPs in katG, rpsL and cysA that were associated with

264 antibiotic resistance, were found in the 38765 strain only. In addition, there were three

265 non-synonymous SNPs in the genes pta, eis and lipU present in 38765. On the other

266 hand, the synonymous SNP at the cysA3 genes was found in 38088 strain only. Exactly

267 the same synonymous SNP at cysA3 was found in non-clonally distributed Beijing

268 strains analyzed in a previous study (28) and in a modern Beijing strains from

269 Guatemala (38).

Except the frameshift deletion at the position 434226, all the remaining SNPs

271 previously reported as exclusively present in the Beijing-like strains from Colombia (32)

272 were also found in the MDR-TB Beijing strain 38765 that was subjected to WGS in this

273 study (Table 2). Instead of the deletion at the position 434226, our Beijing MDR-TB

274 strain (38765) also had a deletion just in the next nucleotide position (434227) (Table 3).

275 For the FS Beijing strain (38088), only one mutation at the Rv2308 gene was shared

276 with another Beijing-like strains from Colombia as well as with 38765 (Table 3). Rv2308

277 encodes for a conserved hypothetical protein that may act as a transcriptional regulator 
278 (40). Importantly, this SNP found in Rv2308 has not previously identified in other Beijing

279 strains worldwide (28).

We acknowledge several limitations in the present study. The study design

281 included convenience sampling with a limited number of Beijing isolates that were

282 available in a repository in Colombia. Nevertheless, the samples included all Beijing

283 strains found in that setting during a 10 years period. Additionally, the repository was

284 built over the years with the cultures successfully sent to our institution for surveillance

285 purposes.

Another potential limitation of our study is related to the selection of SNPs to

consensus on which SNPs are most informative and reliable to accurately describe the

295 locally and worldwide circulating Beijing strains, but will not be readily available in all 296 settings. 
301 local Mtb structure remains important in understanding the epidemiology of MDR-TB

302 and facilitate the development of control strategies against (MDR) TB.

\section{Acknowledgements}

304

305 whole genome sequencing analysis.

\section{Author contributions}

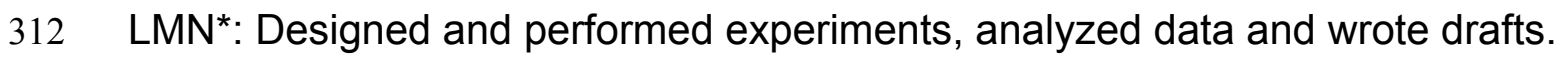

313 BEF*: Designed and performed experiments, analyzed data and wrote drafts.

314 GD: Conducted laboratory analysis and analyzed data,

315 JdB: Conducted laboratory analysis, reviewed and edited drafts.

316 RA: Designed and performed experiments, analyzed data, reviewed and edited drafts.

317 DvS: Designed and performed experiments, analyzed data, reviewed and edited drafts. 


\section{References}

321 1. Glynn JR, Whiteley J, Bifani PJ, Kremer K, van Soolingen D. Worldwide

322 occurrence of Beijing/W strains of Mycobacterium tuberculosis: a systematic review.

323 Emerg Infect Dis. 2002;8(8):843-9.

324 2. Parwati I, van Crevel R, van Soolingen D. Possible underlying mechanisms for

325 successful emergence of the Mycobacterium tuberculosis Beijing genotype strains.

326 Lancet Infect Dis. 2010;10(2):103-11.

327 3. Buu TN, Huyen MN, Lan NT, Quy HT, Hen NV, Zignol M, et al. The Beijing

328 genotype is associated with young age and multidrug-resistant tuberculosis in rural

329 Vietnam. Int J Tuberc Lung Dis. 2009;13(7):900-6.

330 4. European Concerted Action on New Generation Genetic Markers and

331 Techniques for the Epidemiology and Control of Tuberculosis. Beijing/W genotype

332 Mycobacterium tuberculosis and drug resistance. Emerg Infect Dis. 2006;12(5):736-43.

333 5. World, Health, Organization. Global Tuberculosis Report 2017.

334 6. Bifani PJ, Mathema B, Kurepina NE, Kreiswirth BN. Global dissemination of the

335 Mycobacterium tuberculosis W-Beijing family strains. Trends Microbiol. 2002;10(1):45-

33652.

337 7. Shitikov E, Kolchenko S, Mokrousov I, Bespyatykh J, Ischenko D, llina E, et al.

338 Evolutionary pathway analysis and unified classification of East Asian lineage of

339 Mycobacterium tuberculosis. Sci Rep. 2017;7(1):9227. 
340 8. Mokrousov I, Ly HM, Otten T, Lan NN, Vyshnevskyi B, Hoffner S, et al. Origin

341 and primary dispersal of the Mycobacterium tuberculosis Beijing genotype: clues from

342 human phylogeography. Genome Res. 2005;15(10):1357-64.

343 9. Iwamoto T, Grandjean L, Arikawa K, Nakanishi N, Caviedes L, Coronel J, et al.

344 Genetic diversity and transmission characteristics of Beijing family strains of

345 Mycobacterium tuberculosis in Peru. PLoS One. 2012;7(11):e49651.

346 10. Liu Q, Luo T, Dong X, Sun G, Liu Z, Gan M, et al. Genetic features of

347 Mycobacterium tuberculosis modern Beijing sublineage. Emerg Microbes Infect.

$348 \quad 2016 ; 5: e 14$.

349 11. de Keijzer J, de Haas PE, de Ru AH, van Veelen PA, van Soolingen D.

350 Disclosure of selective advantages in the "modern" sublineage of the Mycobacterium

351 tuberculosis Beijing genotype family by quantitative proteomics. Mol Cell Proteomics.

$352 \quad 2014 ; 13(10): 2632-45$.

353 12. Comas I, Coscolla M, Luo T, Borrell S, Holt KE, Kato-Maeda M, et al. Out-of-

354 Africa migration and Neolithic coexpansion of Mycobacterium tuberculosis with modern

355 humans. Nat Genet. 2013;45(10):1176-82.

356 13. Ribeiro SC, Gomes LL, Amaral EP, Andrade MR, Almeida FM, Rezende AL, et

357 al. Mycobacterium tuberculosis strains of the modern sublineage of the Beijing family

358 are more likely to display increased virulence than strains of the ancient sublineage. J

359 Clin Microbiol. 2014;52(7):2615-24.

360 14. Ritacco V, López B, Cafrune PI, Ferrazoli L, Suffys PN, Candia N, et al.

361 Mycobacterium tuberculosis strains of the Beijing genotype are rarely observed in

362 tuberculosis patients in South America. Mem Inst Oswaldo Cruz. 2008;103(5):489-92. 
363 15. Laserson KF, Osorio L, Sheppard JD, Hernández H, Benitez AM, Brim S, et al.

364 Clinical and programmatic mismanagement rather than community outbreak as the

365 cause of chronic, drug-resistant tuberculosis in Buenaventura, Colombia, 1998. Int J

366 Tuberc Lung Dis. 2000;4(7):673-83.

367 16. Abadía E, Sequera M, Ortega D, Méndez MV, Escalona A, Da Mata O, et al.

368 Mycobacterium tuberculosis ecology in Venezuela: epidemiologic correlates of common

369 spoligotypes and a large clonal cluster defined by MIRU-VNTR-24. BMC Infect Dis.

$370 \quad 2009 ; 9: 122$.

371 17. Candia N, Lopez B, Zozio T, Carrivale M, Diaz C, Russomando G, et al. First

372 insight into Mycobacterium tuberculosis genetic diversity in Paraguay. BMC Microbiol.

$373 \quad 2007 ; 7: 75$.

374 18. Jiménez P, Calvopiña K, Herrera D, Rojas C, Pérez-Lago L, Grijalva M, et al.

375 [Identification of the Mycobacterium tuberculosis Beijing lineage in Ecuador]. Biomedica.

$376 \quad 2017 ; 37(2): 233-7$.

377 19. Ferro BE, Nieto LM, Rozo JC, Forero L, van Soolingen D. Multidrug-resistant

378 Mycobacterium tuberculosis, Southwestern Colombia. Emerg Infect Dis.

$3792011 ; 17(7): 1259-62$.

380 20. Nieto LM, Ferro BE, Villegas SL, Mehaffy C, Forero L, Moreira C, et al.

381 Characterization of extensively drug-resistant tuberculosis cases from Valle del Cauca,

382 Colombia. J Clin Microbiol. 2012;50(12):4185-7.

383 21. Villegas SL, Ferro BE, Perez-Velez CM, Moreira CA, Forero L, Martínez E, et al.

384 High initial multidrug-resistant tuberculosis rate in Buenaventura, Colombia: a public-

385 private initiative. Eur Respir J. 2012;40(6):1569-72. 
22. Brudey K, Driscoll JR, Rigouts L, Prodinger WM, Gori A, Al-Hajoj SA, et al. Mycobacterium tuberculosis complex genetic diversity: mining the fourth international spoligotyping database (SpolDB4) for classification, population genetics and epidemiology. BMC Microbiol. 2006;6:23.

23. Kent PT, Kubica GP. Public Health Mycobacteriology. A guide for the level III laboratory. U.S Department of Health and Human Services PHS, Centers for Disease Control CDC, editor. Atlanta, GA1985.

24. Kamerbeek J, Schouls L, Kolk A, van Agterveld M, van Soolingen D, Kuijper S, et al. Simultaneous detection and strain differentiation of Mycobacterium tuberculosis for diagnosis and epidemiology. J Clin Microbiol. 1997;35(4):907-14.

25. Supply P, Allix C, Lesjean S, Cardoso-Oelemann M, Rüsch-Gerdes S, Willery E, et al. Proposal for standardization of optimized mycobacterial interspersed repetitive unit-variable-number tandem repeat typing of Mycobacterium tuberculosis. J Clin Microbiol. 2006;44(12):4498-510.

26. van Embden JD, Cave MD, Crawford JT, Dale JW, Eisenach KD, Gicquel B, et al. Strain identification of Mycobacterium tuberculosis by DNA fingerprinting: recommendations for a standardized methodology. J Clin Microbiol. 1993;31(2):406-9.

27. de Beer JL, Ködmön C, van Ingen J, Supply P, van Soolingen D. Second worldwide proficiency study on variable number of tandem repeats typing of Mycobacterium tuberculosis complex. Int J Tuberc Lung Dis. 2014;18(5):594-600.

28. Schürch AC, Kremer K, Hendriks AC, Freyee B, McEvoy CR, van Crevel R, et al. SNP/RD typing of Mycobacterium tuberculosis Beijing strains reveals local and worldwide disseminated clonal complexes. PLoS One. 2011;6(12):e28365. 
29. Bergval I, Sengstake S, Brankova N, Levterova V, Abadía E, Tadumaze N, et al. Combined species identification, genotyping, and drug resistance detection of Mycobacterium tuberculosis cultures by MLPA on a bead-based array. PLoS One. 2012;7(8):e43240.

30. Sengstake S, Bablishvili N, Schuitema A, Bzekalava N, Abadia E, de Beer J, et al. Optimizing multiplex SNP-based data analysis for genotyping of Mycobacterium tuberculosis isolates. BMC Genomics. 2014;15:572.

31. Banerjee R, Allen J, Westenhouse J, Oh P, Elms W, Desmond E, et al. Extensively drug-resistant tuberculosis in california, 1993-2006. Clin Infect Dis. $2008 ; 47(4): 450-7$

32. Rodríguez-Castillo JG, Pino C, Niño LF, Rozo JC, Llerena-Polo C, Parra-López CA, et al. Comparative genomic analysis of Mycobacterium tuberculosis Beijing-like strains revealed specific genetic variations associated with virulence and drug resistance. Infect Genet Evol. 2017;54:314-23.

33. Ferro BE, García PK, Nieto LM, van Soolingen D. Predictive value of molecular drug resistance testing of Mycobacterium tuberculosis isolates in Valle del Cauca, Colombia. J Clin Microbiol. 2013;51(7):2220-4.

34. Luo T, Zhao M, Li X, Xu P, Gui X, Pickerill S, et al. Selection of mutations to detect multidrug-resistant Mycobacterium tuberculosis strains in Shanghai, China. Antimicrob Agents Chemother. 2010;54(3):1075-81.

35. Devaux I, Manissero D, Fernandez de la Hoz K, Kremer K, van Soolingen D, network E. Surveillance of extensively drug-resistant tuberculosis in Europe, 2003-2007. Euro Surveill. 2010;15(11). 
432 36. Café Oliveira LN, Muniz-Sobrinho JaS, Viana-Magno LA, Oliveira Melo SC,

433 Macho A, Rios-Santos F. Detection of multidrug-resistant Mycobacterium tuberculosis

434 strains isolated in Brazil using a multimarker genetic assay for katG and rpoB genes.

435 Braz J Infect Dis. 2016;20(2):166-72.

436 37. Sheen P, Couvin D, Grandjean L, Zimic M, Dominguez M, Luna G, et al. Genetic

437 diversity of Mycobacterium tuberculosis in Peru and exploration of phylogenetic

438 associations with drug resistance. PLoS One. 2013;8(6):e65873.

439 38. Saelens JW, Lau-Bonilla D, Moller A, Medina N, Guzmán B, Calderón M, et al.

440 Whole genome sequencing identifies circulating Beijing-lineage Mycobacterium

441 tuberculosis strains in Guatemala and an associated urban outbreak. Tuberculosis

442 (Edinb). 2015;95(6):810-6.

443 39. Villellas C, Aristimuño L, Vitoria MA, Prat C, Blanco S, García de Viedma D, et al.

444 Analysis of mutations in streptomycin-resistant strains reveals a simple and reliable

445 genetic marker for identification of the Mycobacterium tuberculosis Beijing genotype. J

446 Clin Microbiol. 2013;51(7):2124-30.

447 40. Camus JC, Pryor MJ, Médigue C, Cole ST. Re-annotation of the genome

448 sequence of Mycobacterium tuberculosis H37Rv. Microbiology. 2002;148(Pt 10):2967-

44973. 


3143

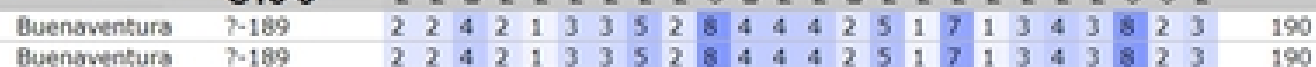
$\begin{array}{lllllllllllllllllllllllllllll}\text { Buenaventura } & 7.189 & 2 & 2 & 4 & 2 & 1 & 3 & 3 & 5 & 2 & 8 & 4 & 4 & 4 & 2 & 5 & 1 & 7 & 1 & 3 & 4 & 3 & 8 & 2 & 3 & 190 \\ \text { Buenaventura } & 7.189 & 2 & 2 & 2 & 4 & 2 & 1 & 3 & 3 & 5 & 2 & 8 & 4 & 4 & 4 & 2 & 5 & 1 & 7 & 1 & 3 & 4 & 3 & 8 & 2 & 3 & 190\end{array}$ Buenaventurs $\quad 7-189$ $\begin{array}{lllllllllllllllllllllllllll}2 & 2 & 4 & 2 & 1 & 3 & 3 & 5 & 2 & 8 & 4 & 4 & 4 & 2 & 5 & 1 & 7 & 1 & 3 & 4 & 3 & 8 & 2 & 3 & 190 \\ 2 & 4 & 1 & 4 & 4 & 2 & 5 & 1 & 7 & 1 & 3 & 4 & 3 & 8 & 2 & 3 & 190\end{array}$ evenaventura $\quad 7-189$ $\begin{array}{lllllllllllllllllllllllllll}2 & 2 & 4 & 2 & 1 & 3 & 3 & 5 & 2 & 9 & 4 & 4 & 4 & 2 & 5 & 1 & 7 & 1 & 3 & 4 & 3 & 8 & 2 & 3 & 190 \\ 2 & 2 & 4 & 2 & 1 & 3 & 3 & 5 & 2 & 9 & 4 & 4 & 4 & 2 & 5 & 1 & 7 & 1 & 3 & 4 & 3 & 8 & 2 & 3 & 190\end{array}$ ouenaventurs $\quad 7-189$ Quenaventurs 7.189 $\begin{array}{lllllllllllllllllllllllllll}2 & 2 & 4 & 2 & 1 & 3 & 3 & 5 & 2 & 9 & 4 & 4 & 4 & 2 & 5 & 1 & 7 & 1 & 3 & 4 & 3 & 8 & 2 & 3 & 190\end{array}$ Quenaventura 7.189 $\begin{array}{lllllllllllllllllllllllll}2 & 2 & 4 & 2 & 1 & 3 & 3 & 5 & 2 & 9 & 4 & 4 & 4 & 2 & 5 & 1 & 7 & 1 & 3 & 4 & 3 & 8 & 2 & 3 & 190 \\ 2 & 2 & 4 & 2 & 1 & 3 & 5 & 5 & 9 & 4 & 4 & 4 & 4 & 2 & 3 & 190\end{array}$ Auenaventura $\begin{array}{lllllllllllllllllllllllllll}2 & 2 & 4 & 2 & 1 & 3 & 3 & 5 & 2 & 9 & 4 & 4 & 4 & 2 & 5 & 1 & 7 & 1 & 3 & 4 & 3 & 8 & 2 & 3 & 190 \\ 2 & 2 & 4 & 2 & 1 & 3 & 3 & 5 & 2 & 9 & 4 & 4 & 4 & 2 & 5 & 1 & 7 & 1 & 3 & 4 & 3 & 8 & 2 & 3 & 190\end{array}$ Buenaventuras 7.189 Buenaventurs 7.189 $\begin{array}{lllllllllllllllllllllllll}2 & 4 & 2 & 1 & 3 & 3 & 5 & 2 & 9 & 4 & 4 & 4 & 2 & 5 & 1 & 7 & 1 & 3 & 4 & 3 & 8 & 2 & 3 & 190\end{array}$ Buenaventurs? $?+189$ Buenaventura 7-189 Buenaventura $7-189$ Buenaventurs Buenaventurs Buenaventura Buenaventura Buenoventura Buenaventura \begin{tabular}{llllllllllllllllllllllllll}
2 & 2 & 4 & 2 & 1 & 3 & 3 & 5 & 2 & 9 & 4 & 4 & 4 & 2 & 5 & 1 & 7 & 1 & 3 & 4 & 3 & 8 & 2 & 3 & 190 \\
\hline & 2 & 4 & 2 & 1 & 3 & 3 & 5 & 2 & 9 & 4 & 4 & 4 & 2 & 5 & 1 & 7 & 1 & 3 & 4 & 3 & 8 & 2 & 3 & 1900
\end{tabular} $\begin{array}{llllllllllllllllllllllllll}2 & 2 & 4 & 2 & 1 & 3 & 3 & 5 & 2 & 9 & 4 & 4 & 4 & 2 & 5 & 1 & 7 & 1 & 3 & 4 & 3 & 8 & 2 & 3 & 190\end{array}$ \begin{tabular}{lllllllllllllllllllllllllll}
2 & 2 & 4 & 2 & 1 & 3 & 3 & 5 & 2 & 9 & 4 & 4 & 4 & 2 & 5 & 1 & 7 & 1 & 3 & 4 & 3 & 8 & 2 & 3 & 190 \\
\hline & 2 & 2 & 4 & 2 & 3 & 3 & 5 & 2 & 9 & 4 & 4 & 4 & 2 & 5 & 1 & 7 & 1 & 3 & 4 & 3 & 8 & 2 & 3 & & 1900
\end{tabular} $\begin{array}{llllllllllllllllllllllllll}2 & 2 & 4 & 2 & 1 & 3 & 3 & 5 & 2 & 9 & 4 & 4 & 4 & 2 & 5 & 1 & 7 & 1 & 3 & 4 & 3 & 8 & 2 & 3 & 190 \\ 2 & 2 & 4 & 2 & 1 & 3 & 3 & 5 & 2 & 9 & 4 & 4 & 4 & 2 & 5 & 1 & 7 & 1 & 3 & 4 & 3 & 8 & 2 & 3 & & 190\end{array}$ $\begin{array}{llllllllllllllllllllllllll}2 & 2 & 4 & 2 & 1 & 3 & 3 & 5 & 2 & 9 & 4 & 4 & 4 & 2 & 5 & 1 & 7 & 1 & 3 & 4 & 3 & 8 & 2 & 3 & 190\end{array}$

Buenaventura

$7-189$ Buenoventura $7-189$ Buenaventura Cali Buenaventura Buenaventura ND Buenaventura Buenaventurs Buenaventurs
Buenawentura
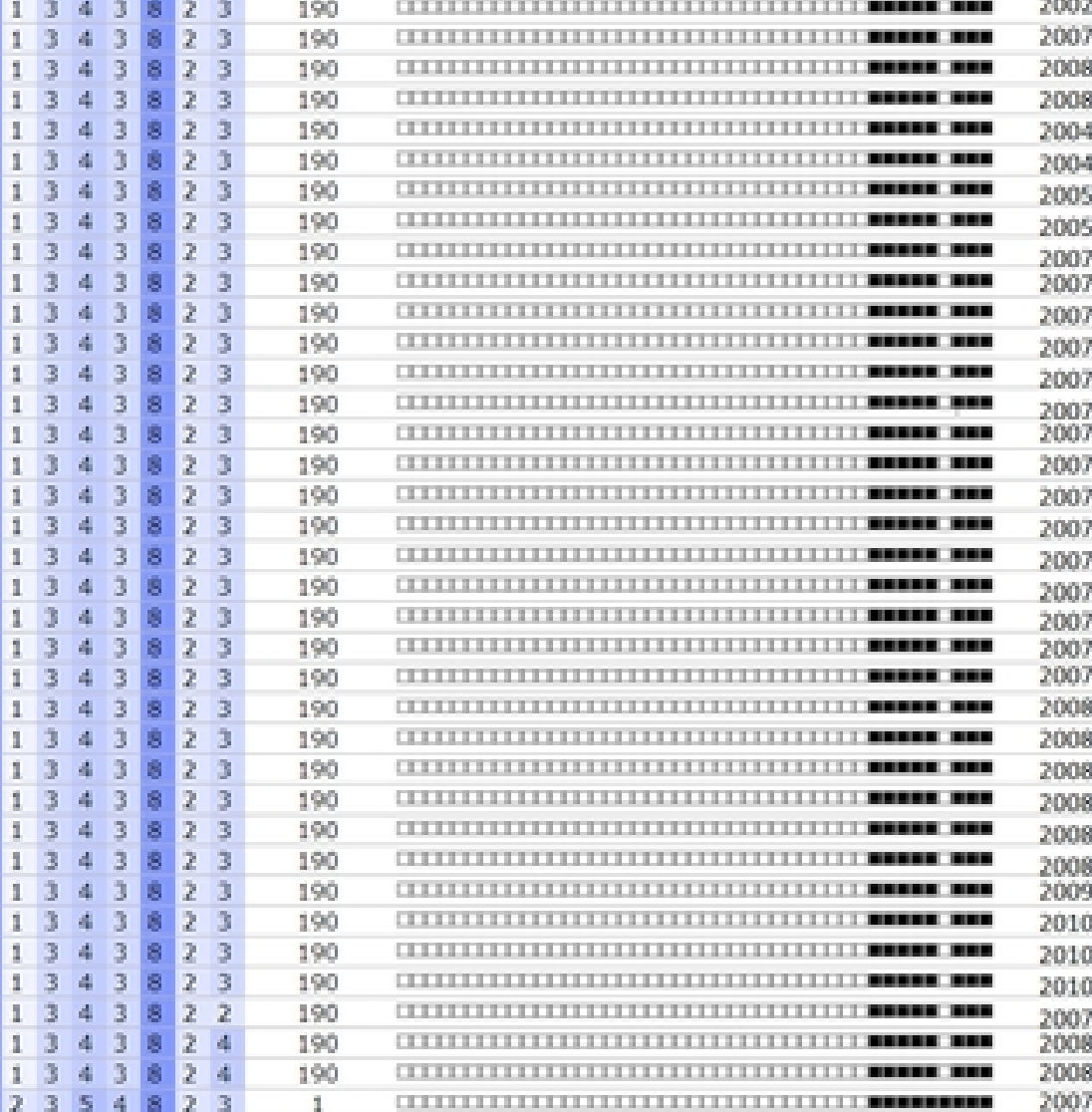
2002 DSP 7117
$3846-220$

$\begin{array}{llllllllllllllllllllllllll}11177 & 2 & 2 & 4 & 2 & 1 & 3 & 3 & 5 & 2 & 9 & 4 & 4 & 4 & 2 & 5 & 1 & 7 & 1 & 3 & 4 & 3 & 8 & 2 \\ 3846-229 & 2 & 4 & 4 & 2 & 3 & 3 & 3 & 5 & 2 & 6 & 4 & 4 & 4 & 2 & 5 & 1 & 7 & 2 & 3 & 5 & 4 & 8 & 2\end{array}$

[IIIIIIII)

romen

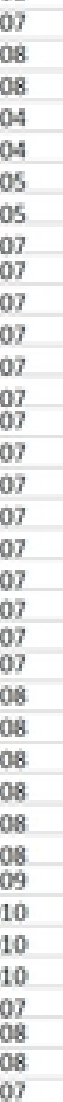

